\title{
Resource Allocation for Device-to-Device Communications Underlaying Cellular Networks
}

\author{
Tao $\mathrm{He}^{1}$, Xinquan $\mathrm{Ye}^{2}$ and Lieying Luo ${ }^{3}$ \\ ${ }^{1}$ Oujiang college of Wenzhou University, Wenzhou325035, P.R.CHINA \\ ${ }^{2}$ College of Mechnical \& Electrical Engineering, Wenzhou University, \\ Wenzhou325035, P.R.CHINA \\ ${ }^{3}$ City college of Wenzhou University, Wenzhou325035, P.R.CHINA \\ luolieying@126.com
}

\begin{abstract}
Device-to-Device (D2D) communications potentially can improve spectrum efficiency because of resource spatial reuse. There are two possibilities for D2D resource allocation: contention-based resource allocation and scheduling-based resource allocation. In this paper, these resource allocation schemes are analyzed. Then, we propose a scheduling-based resource allocation that has the advantage of low scheduling overhead in addition to providing a common design for under the in-network coverage and out-of-network coverage. Simulation results show that the proposed algorithm can increase system throughput by exploring spatial reuse gain and maintain good proportional fairness among D2D communication pairs.
\end{abstract}

Keywords: Device-to-Device, Resource Allocation, resource spatial reuse, low overhead

\section{Introduction}

Recently, a new communication method called Device-to-Device (D2D) communication has been paid more and more attentions. The concept of D2D communication as an underlay to a cellular network is that user equipments (UEs) can directly communicate with each other while remaining controlled by evolved NodeBs (eNBs) [1]. Different from Bluetooth and WiFi-Direct, D2D communication is working in a liscensed spectra, which is more reliable and secure. Besides that, saving power consumption, releasing eNBs loads and improving spectrum efficiency are also advantages for D2D communication, so it has a promising commercial prospect [2].

D2D communication in cellular networks consists of two phases [3]. The first phase is D2D peer discovery and the second phase is data transmission [4]. This paper will focus on the second phase. In the data transmission phase, D2D link may be interfered by cellular UEs or by other D2D links. So a reasonable wireless resource allocation method should be applied to mitigate interference so as to guarantee the quality of data transmission [5]. This paper mainly studies the wireless resource allocation method for D2D communications in cellular networks [6].

For D2D Communication, there are two candidates for the resource allocation [7]:

Scheme 1: contention-based resource allocation: the devices contend for resources for D2D transmission. 
- Scheme 2: scheduling-based resource allocation: the network (eNB as master controller for in-network scenarios, and a group master for out-of-network scenarios) allocates resources for the D2D link.

In this paper, these resource allocation schemes are analyzed. The analysis shows the scheduling-based scheme provides deployment advantages over the contention-based resource allocation. We thus suggest using the eNB as master controller for in-network scenarios, and a group master for out-of-network scenarios. Then, we propose a scheduling-based resource allocation that has the advantage of low scheduling overhead in addition to providing a common design for under the in-network coverage and out-ofnetwork coverage.

\section{Contention-Based vs. Scheduling-Based Resource Allocation}

\subsection{In-Network Coverage}

2.1.1. Contention-Based Scheme: An advantage of such a contention-based scheme is no scheduling overhead. In addition, the same scheme can be applied as the number of UEs increases. However, resource collision among transmitting D2D UEs can degrade the SINR at a particular device [8]. Thus, a collision management scheme is needed (similar to 802.11 peer-to-peer transmission mode). In addition, contention-based schemes may lead to a large delay in delay-sensitive applications like voice. Reference Error! Reference source not found. shows that the throughput associated with a contention-based scheme, compared to a scheduling-based scheme, deteriorates significantly as the number of transmitters increases.

2.1.2. Scheduling-Based Scheme: Given that the eNB has more information than a UE, minimizing interference (consequently higher throughput and better QoS control) is much easier than scheme 1 . In addition, this scheme requires minimal standard changes for both RAN1 and RAN2. Note that a scheduling-based scheme introduces some overhead, which can be reduced by semi-persistent scheduling [10]. Considering the advantages of the scheduling-based scheme, we propose, for in-network coverage, the resource allocation is managed by the network (eNB).

\subsection{Out-of-Network Coverage}

2.2.1. Contention-Based Scheme: In this type of resource assignment, UEs listen all the time. When a UE wants to transmit data, it sends a signal in an asynchronous manner. The UE receiving this signal then sends a message to indicate its proximity. For data transmission, a handshake process is used before actual data transmission. Conceptually, this scheme is similar to the 802.11 peer-to-peer transmission mode. Moreover, for groupcast communication, a competition process could be used before data transmission, and scheduling priority should be considered in competition process. However, in order to minimize standardization efforts, the same numerology as for LTE, or multiple of these values, should be used whenever possible [11].

This scheme is a substantial shift from the traditional LTE architecture and represents a communication mode close to what can be achieved with 802.11 systems. While such a resource assignment is relatively simple to design, it would require a major standardization effort. Furthermore, its performance does not scale well when the number of UEs is relatively large (larger than 10).

2.2.2. Scheduling-Based Scheme: In the scheduling-based resource assignment scheme, in-network and out-of-network cases can be treated similarly. This scheme requires minimal standard changes, at the cost of increased power consumption for the 
group master since when out-of-coverage, the devices need to operate in an asynchronous mode. To further simplify the group master and to ensure a fair scheduler or for emergency cases, scheduling priority could be reported to the controller. The group master can provide the minimal control information necessary to operate the direct communication link. It also provides a joint solution for in-network and out-of-network in terms of transmission parameters [12]. In case of having multiple groups, the performance of this scheme will be improved if resources can be coordinated among group masters.

The scheduling-based scheme provides the advantage of a common design for innetwork and out-of-network; thus limits the standardization effort. Furthermore if desirable, it is scalable. Consequently, we propose the following: For out-of-network communication, one UE acts as a group master for scheduling purpose. This solution enables a common design for in-network and out-of-network communication. If in outof-network-coverage, the master controller can be selected via a contention-based algorithm.

We have summarized the benefits of contention-based and scheduling-based schemes in Table 1.

Table 1. Contention-Based and Scheduling-Based D2D Resource Allocations: Benefits and Drawbacks

\begin{tabular}{|c|c|c|}
\hline & $\begin{array}{l}\text { Contention-based } \\
\text { Scheme }\end{array}$ & Scheduling-based Scheme \\
\hline Benefit & $\begin{array}{ll}\text { - } & \text { No } \\
& \text { overhead }\end{array}$ & $\begin{array}{l}\text { - Good performance due to interference } \\
\text { management gain } \\
\text { - Common design principles for in- and out-of- } \\
\text { network coverage } \\
\text { - Consistent with existing cellular communication } \\
\text { structure, hence less standard impact }\end{array}$ \\
\hline Drawback & 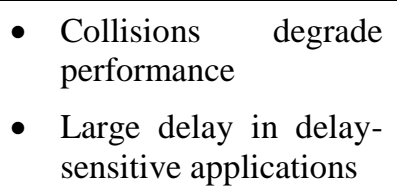 & $\begin{array}{l}\text { - Scheduling overhead can increase with } \\
\text { increasing number of UEs, but can be reduced } \\
\text { with semi-persistent scheduling }\end{array}$ \\
\hline
\end{tabular}

\section{Proposed Resource Allocation Design}

Error! Reference source not found. shows a two-step semi-static resource assignment algorithm. In the first step, the eNB (or group master) configures two D2Dcapable devices with a set of radio resources, such as frequency subcarriers and/or time slots, where the two devices can communicate with each other directly. This is a highlevel configuration in the sense that the eNB neither establishes transmission parameters nor controls which packets are sent [13]. The eNB does not send downlink control information (DCI) for each packet. In the second stage, a "fine" resource allocation, containing packet and MCS information, is sent by the devices engaged in D2D communication. 


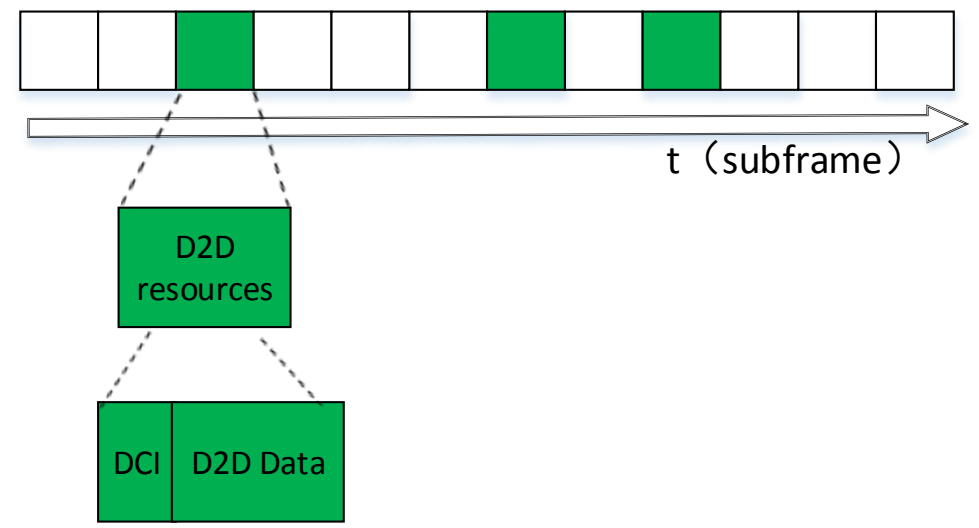

\section{Figure 1. Two-Step Resource Assignment for Direct Communication}

For frequency allocation, the first step can be based on the existing Semi Persistent Signaling (SPS). For the second step, a physical layer control message such as a simplified DCI is added for each block transmitted from one D2D device to the other, as shown in Error! Reference source not found.. Note that the control information may be located elsewhere in the subframe Error! Reference source not found..

\section{Dontrol}

DATA

Figure 2. Direct Communication Packet with its $\mathrm{DCl}$

For the time allocation, one of the key aspects to address is groupcast transmission. The eNB needs to:

a) Decide which subframes are allocated to this particular D2D group (Error! Reference source not found. top)

b) Within the group, decide which device is transmitting on a given subframe (Error! Reference source not found. middle)

With this information, each device can be notified when to transmit and deduce when to receive (Error! Reference source not found. bottom). For example, the eNB can indicate the transmission subframes using a bitmap.
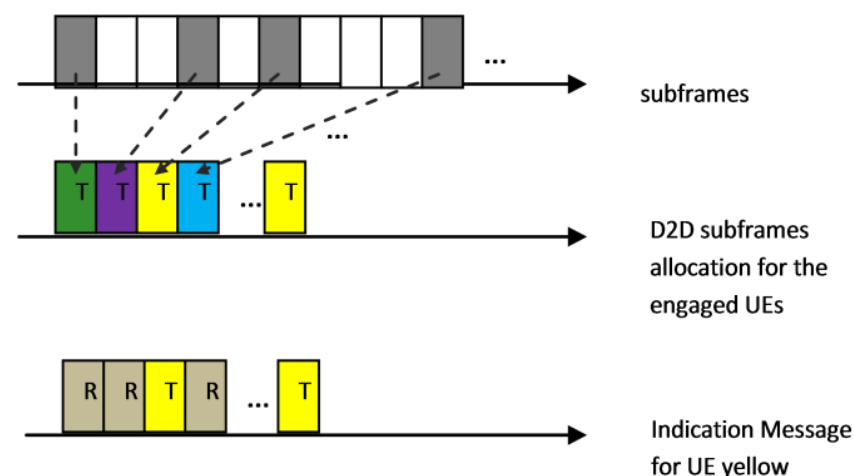

Figure 3. Subframe Allocation for Direct Communication. Different UEs are Shown by Different Colors. 
We propose a Semi-static scheduling-based resource assignment design. Each member of the D2D group is allocated resources by the eNB. The resources are allocated in a semi-persistent manner.

\section{Simulation and Performance Analysis}

Some simulation analyses of Contention-based scheme and Scheduling-based scheme for both VoIP and full buffer traffic mode are provided. For VoIP traffic, three resource allocation options are considered:

Option 1: Contention-based scheme

Option 2: Scheduling-based scheme

Option 3: Proposed resource allocation design

In Fig. 4, CDFs of UE-UE distances are shown where only those links that are not in outage would be counted. Per link outage is defined as the probability of VoIP packet drop is higher than $2 \%$. The packet drop is either due to the unsuccessful decoding, or transmission delay is more than $200 \mathrm{~ms}$. Fig. 5 shows the CDFs of packet drop per link under the three options. As shown in Figs 5-6, because of the light traffic load in VoIP simulation setup, e.g., 3 broadcasting UEs per cell with $10 \mathrm{MHz}$, three resource allocation schemes have almost the same performance. The proposed resource allocation design performs slightly better than the others.

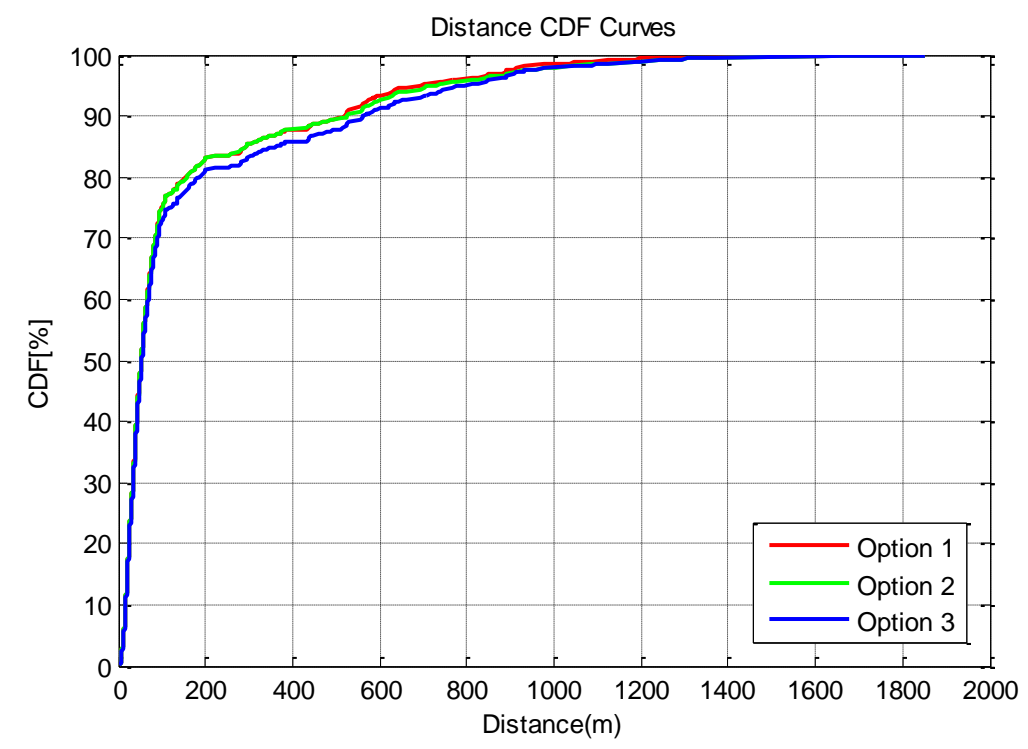

Figure 4. D2D VolP Broadcast Coverage Distributions for Different Resource Allocations 


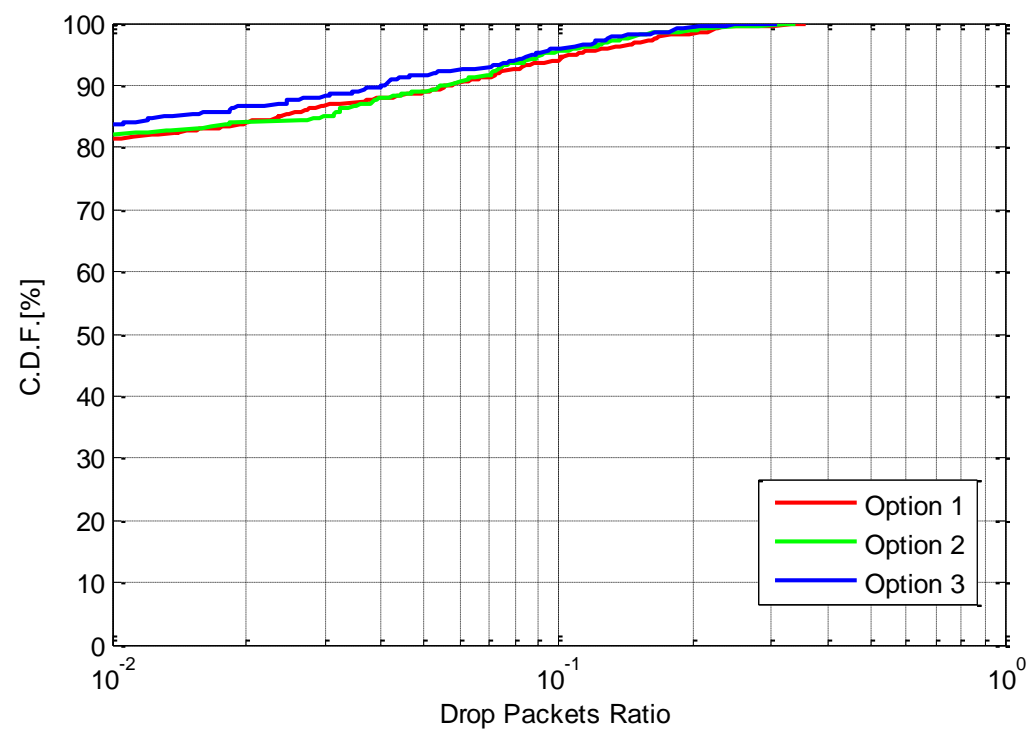

Figure 5. Packet Drop Ratio Distribution of D2D VoIP Broadcast for
Different Resource Allocations

\section{Conclusion}

By analyzing resource allocation for device-to-device communications underlaying cellular networks. The processes of Contention-based scheme and Scheduling-based scheme are discussed in detail. This paper proposes a scheduling-based resource allocation that has the advantage of low scheduling overhead in addition to providing a common design for under the in-network coverage and out-of-network coverage. We propose: Centralized scheduling may be supported when in network coverage or if cluster head can reliably fulfill the task of eNB. Distributed resource allocation should be supported when out of network coverage and no $\mathrm{CH}$ available. Time and frequency diversity is crucial to VoIP performance of D2D broadcast. Narrowband D2D broadcast communication, e.g., 1 or 2 PRBs, is preferred. Finally, simulation and comparison analysis of this scheme. The simulation results show that the improved design can satisfy the resource allocation for device-to-device communications requirements.

\section{Acknowledgements}

This work was supported by Natural Science Foundation of China (11372229, 71271156, 71301120), and Education Department of Zhejiang Province (Z201120727, Y201224800). Science Technology Department of Zhejiang Province (2012C14026, 2012C21050). 


\section{References}

[1] Y. Jeong, M. Kim and M. Y. Chung, “ Frequency-Domain Packet Scheduling for Low PAPR in 3GPP LTE Uplink", International Journal of Smart Home, vol. 7, no. 1, (2013),

[2] M. Belleschi, G. Fodor and A. Abrardo, "Performance analysis of a distributed resource allocation scheme for D2D communications", [C]//GLOBECOM Workshops (GC Wkshps), 2011 IEEE. IEEE, (2011), pp.358-362.

[3] P. Phunchongharn, E. Hossain and D I. Kim, "Resource allocation for device-to-device communications underlaying LTE-advanced networks", [J]. Wireless Communications, IEEE, vol. 20, no. 4, (2013), pp. 91-100.

[4] Y. Pei and Y. C. Liang, "Resource allocation for device-to-device communications overlaying two-way cellular networks", [J]. Wireless Communications, IEEE Transactions on, vol. 12, no. 7, (2013), pp. 3611-3621.

[5] X. Lin, J. G. Andrews and A. Ghosh, “An overview of 3GPP device-to-device proximity services”, [J]. Communications Magazine, IEEE, (2014), vol. 52, no. 4, pp. 40-48.

[6] H. D. Bae, J. W. Shin and P. J. Song, "Mode Switching for Device-to-Device Communication in LTEA Network", [C]//Proceedings of the 12th International Conference on Advances in Mobile Computing and Multimedia. ACM, (2014), pp.7-10.

[7] S. Sarkar and M. Tijare, "Resource Sharing of Device to Device Communication in Cellular Network[C]//International Journal of Engineering Research and Technology. ESRSA Publications, vol. 4, (2015) January 1, (2015),

[8] S. Maghsudi and S. Stanczak, "Hybrid Centralized-Distributed Resource Allocation for Device-toDevice Communication Underlaying Cellular Networks", [J]. arXiv preprint arXiv:1502.04539, (2015).

[9] J. Gu, H. W. Yoon and J. Lee, "A resource allocation scheme for device-to-device communications using LTE-A uplink resources", [J]. Pervasive and Mobile Computing, (2015).

[10] T. D. Hoang, L. B. Le and T. Le-Ngoc, "Resource allocation for D2D communications under proportional fairness", [C]//Global Communications Conference (GLOBECOM), 2014 IEEE. IEEE, (2015), pp. 1259-1264.

[11] C.Vlachos and V. Friderikos, "Robust randomized resource allocation for Device-to-Device communications", $[\mathrm{C}] / /$ Computer Aided Modeling and Design of Communication Links and Networks (CAMAD), 2014 IEEE 19th International Workshop on. IEEE, (2015), pp. 365-369.

[12] J. Xue and B. Wen, "Research on Resource Allocation for Device-to-Device Communication Underlaying LTE Network”, [J]. Journal of Computational Information Systems, vol. 11, no. 1, (2015), pp. 75-86.

[13] Alamouti S M, Sharafat A R. Resource allocation for energy-efficient device-to-device communication in $4 \mathrm{G}$ networks[C]//Telecommunications (IST), 2014 7th International Symposium on. IEEE, (2014): 1058-1063.

[14] Wang L, Liu L, Cao X, et al. Sociality-aware resource allocation for device-to-device communications in cellular networks[J]. IET Communications, (2015).

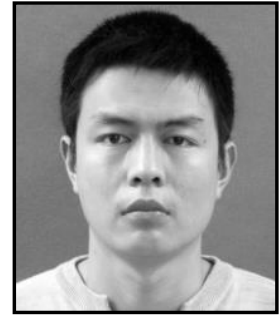

Tao He received the BE degree in computer science from WuHan University of Science and Technology, China, in 2005, the ME degree in mechanical engineering from WuHan University of Technology, China, in 2008. He is currently a Lecturer in the mechanical engineering, the WenZhou University, China. His current research interests are in the areas of Intelligent detection and control, the complex system optimization. He has worked intensively on designing, analyzing and evaluating practical algorithms with the theoretical basis, as well as building prototype systems. His research work is supported by several ZheJiang Science and technology grants.

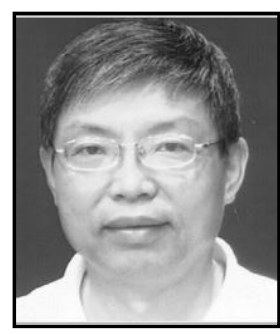

Xianquan Ye received the $\mathrm{BE}$ degree in mechanical engineering from TianJin University of of Science and Technology, China, in 1982. His current research interests are in the areas of High/Low voltage electrical equipment system, the complex system optimization, stochastic optimization. His research work is supported by several ZheJiang Science and technology grants. 


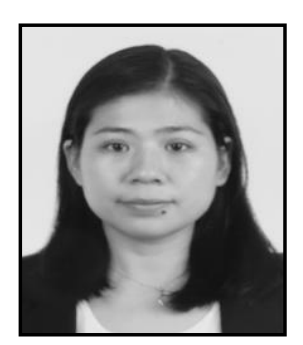

Lieying Luo received the BE degree in Mechanical design manufacturing and automation from WuHan University of Technology, China, in 2004, the ME degree in Logistics Engineering from WuHan University of Technology, China, in 2007. Her current research interests are in the areas of Intelligent detection and control, the Logistics system optimization. Her research work is supported by several ZheJiang Science and technology grants. 